\title{
An Efficient and Convenient Approach to Synthesis of Tetrahydrobenzo $[b]$ pyran Derivatives Using Tetrabutylammonium Bromide as Catalyst
}

\author{
S. GURUMURTHI, V. SUNDARI* AND R. VALLIAPPAN ${ }^{\S}$ \\ Chemistry Division, Faculty of Engineering and Technology, \\ ${ }^{\S}$ Chemistry Wing, DDE, Annamalai University, \\ Annamalai Nagar-608 002, India. \\ sgmurthi1973@yahoo.com
}

Received 30 April 2009; Accepted 6 June 2009

\begin{abstract}
An efficient and convenient approach to the synthesis of 2-amino-3cyano-4-aryl-7,7-dimethyl-5-oxo-4H-5, 6, 7, 8- tetrahydro benzo[b]pyran derivatives using tetrabutylammonium bromide (TBABr) as the catalyst is described. This method provides several advantages such as neutral conditions, high yields and simple work procedure.
\end{abstract}

Keywords: Tetrahydro benzo[b]pyran, Tetrabutylammonium bromide, Synthesis.

\section{Introduction}

In recent years $4 H$ benzo[b]pyrans and their derivatives have attracted considerable attention due to their wide range of biological activities ${ }^{1}$. Further more these compounds can be employed as pigments, photoactive materials ${ }^{2}$ and they constitute the structural unit of a series of natural products ${ }^{3}$.

The importance of these compounds has led many workers to synthesize them using methods including microwave ${ }^{4}$, electrosynthesis ${ }^{5}$ or by using catalysts like hexadecyltrimethylammonium bromide (HTMAB $)^{6}$, triethylbenzylammonium chloride $(\mathrm{TEBA})^{7},(s)$-proline ${ }^{8}$, perfluorooctanoate $\left[\mathrm{RE}(\mathrm{PFO})_{3}\right]^{9}$ and $N$-methylimidazole ${ }^{10}$.

Each of the above methods has its own merits, while some are plegued by limitation of poor yields, difficult work up and toxic effluents. $\mathrm{TBABr}$ has been used ${ }^{11}$ in many organic reactions as a catalyst. However, the use of $\mathrm{TBABr}$ as catalyst in the synthesis of tetrahydro benzo $[b]$ pyran derivatives has not been reported. In the present investigation we wish to report efficient procedure for the synthesis of tetrahydrobenzo[b]pyrans using an inexpensive and commercially available $\mathrm{TBABr}$ as catalyst. In addition, water was chosen as a green solvent, which also gives the corresponding products in excellent yields. 


\section{Experimental}

\section{Materials and methods}

All the chemicals were purchased from s.d.fine chem. Limited (India) and were used as such without further purification. Melting points were determined by open capillary method and are uncorrected. Infrared (IR) spectra were recorded on Avatar-330 FTIR spectrometer. ${ }^{1} \mathrm{H}$ NMR and ${ }^{13} \mathrm{C}$ NMR spectra were obtained on a Bruker AMXC-500MHz Spectrometer in DMSO. Chemical shifts are expressed in $\delta(\mathrm{ppm})$ values. The following abbreviations were used to indicate the peak multiplicity s-singlet, d-doublet, t-triplet, qquartet, and m-multiplet.

\section{Methods $A$ and $B$}

In a typical procedure, equimolar amounts of arylaldehyde $\mathbf{1}$, malononitrile $\mathbf{2}$ and dimedone (5,5-dimethyl-1,3-cyclohexanedione) $\mathbf{3}$, were mixed with tetrabutylammonium bromide $(10 \mathrm{~mol} \%)$ in $15 \mathrm{~cm}^{3} 90 \%$ of ethanol (method A) or in $15 \mathrm{~cm}^{3}$ water (method B) and refluxed with stirring for the specific time indicated in Table 1. After the completion of the reaction, the mixture was cooled to room temperature and poured into ice (method A) or was kept at refrigerator overnight (method B) to get the crude products. The crude products were purified by recrystallization from ethanol.

Table 1. Synthesis of tetrahydrobenzo $[b]$ pyrans catalyzed by TBABr.

\begin{tabular}{|c|c|c|c|c|c|c|c|c|c|}
\hline \multirow[b]{2}{*}{ 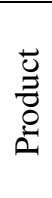 } & \multirow[b]{2}{*}{$\mathrm{Ar}$} & \multicolumn{3}{|c|}{ Time / min } & \multicolumn{3}{|c|}{ Yield, \% } & \multicolumn{2}{|c|}{ M. P / ${ }^{\circ} \mathrm{C}$} \\
\hline & & $\underset{I}{\stackrel{T}{O}}$ & $\begin{array}{l}\text { O } \\
\text { I }\end{array}$ & 节 & $\underset{\text { II }}{\stackrel{T}{0}}$ & 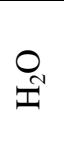 & 节 & 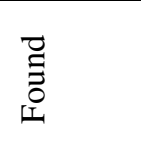 & $\begin{array}{l}\vec{d} \\
\stackrel{0}{0} \\
\text { Q } \\
\text { a }\end{array}$ \\
\hline $4 a$ & $\mathrm{C}_{6} \mathrm{H}_{5}$ & 20 & 60 & 5 & 92 & 88 & 94 & $225-227$ & $226-228^{9}$ \\
\hline $4 b$ & $2-\mathrm{Cl}-\mathrm{C}_{6} \mathrm{H}_{4}$ & 45 & 360 & 30 & 93 & 90 & 90 & $218-220$ & $217-218^{6}$ \\
\hline $4 c$ & $3-\mathrm{Cl}-\mathrm{C}_{6} \mathrm{H}_{4}$ & 60 & 450 & 45 & 90 & 85 & 92 & $235-237$ & - \\
\hline 4d & $4-\mathrm{Cl}-\mathrm{C}_{6} \mathrm{H}_{4}$ & 30 & 120 & 10 & 95 & 91 & 92 & $209-210$ & $209-211^{8}$ \\
\hline $4 e$ & $2-\mathrm{NO}_{2}-\mathrm{C}_{6} \mathrm{H}_{4}$ & 60 & 480 & 40 & 91 & 87 & 92 & $215-217$ & - \\
\hline $4 f$ & $3-\mathrm{NO}_{2}-\mathrm{C}_{6} \mathrm{H}_{4}$ & 90 & 540 & 50 & 90 & 86 & 88 & 207-209 & $208-210^{6}$ \\
\hline $4 g$ & $4-\mathrm{NO}_{2}-\mathrm{C}_{6} \mathrm{H}_{4}$ & 40 & 180 & 25 & 92 & 89 & 91 & $175-177$ & $177-178^{6}$ \\
\hline $4 h$ & $4-\mathrm{Me}-\mathrm{C}_{6} \mathrm{H}_{4}$ & 75 & 440 & 45 & 90 & 85 & 91 & $214-215$ & $214-216^{6}$ \\
\hline $4 \mathbf{i}$ & $\mathrm{N}(\mathrm{Me})_{2}-\mathrm{C}_{6} \mathrm{H}_{4}$ & 95 & 510 & 53 & 92 & 90 & 92 & 197-199 & $198-200^{5}$ \\
\hline $4 j$ & $4-\mathrm{MeO}-\mathrm{C}_{6} \mathrm{H}_{4}$ & 120 & 630 & 48 & 87 & 80 & 90 & $199-200$ & $199-201^{6}$ \\
\hline $4 k$ & $3,4-(\mathrm{MeO})_{2}-\mathrm{C}_{6} \mathrm{H}_{3}$ & 140 & 675 & 55 & 90 & 88 & 91 & $158-160$ & - \\
\hline & $3-\mathrm{Br}-\mathrm{C}_{6} \mathrm{H}_{4}$ & 55 & 340 & 40 & 89 & 90 & 90 & $227-229$ & $225-227^{12}$ \\
\hline & $4-\mathrm{F}-\mathrm{C}_{6} \mathrm{H}_{4}$ & 45 & 300 & 20 & 95 & 91 & 96 & $176-178$ & - \\
\hline $4 n$ & $\mathrm{C}_{6} \mathrm{H}_{5}-\mathrm{CH}=\mathrm{CH}$ & 140 & 720 & 70 & 90 & 90 & 89 & $205-207$ & - \\
\hline 40 & Furfuryl & 95 & 150 & 35 & 90 & 86 & 91 & $217-219$ & $218-220^{13}$ \\
\hline
\end{tabular}

\section{Method C}

A mixture of the reactants as given in methods A and B was grounded for the appropriate time using a dry mortar and pestle. The initial reaction mixture solidified within 5-70 min. The solid was washed with cold water and recrystallized from ethanol. 
2-Amino-3-cyano-5,6,7,8-tetrahydro-7,7-dimethyl-5-oxo-4-phenyl-4H-benzopyran (4a) IR $(\mathrm{KBr}) \mathrm{cm}^{-1} 3396\left(\mathrm{NH}_{2}\right), 3028(\mathrm{C}-\mathrm{H}), 2198(\mathrm{CN}), 1682(\mathrm{C}=\mathrm{O}), 1601(\mathrm{C}=\mathrm{C}) ;{ }^{1} \mathrm{H}$ NMR (DMSO), $\delta$ 7.28-7.07(m, 5H), 6.99(s, 2H, NH $\mathrm{NH}_{2}, 4.14(\mathrm{~s}, 1 \mathrm{H}, \mathrm{H}-4), 2.48(\mathrm{~s}, 2 \mathrm{H}, \mathrm{H}-8)$, 2.22(d, 1H, H-6), 2.08(d, 1H, H-6'), 1.01(s, 3H, $\left.\mathrm{CH}_{3}\right), 0.93\left(\mathrm{~s}, 3 \mathrm{H}, \mathrm{CH}_{3}\right) ;{ }^{13} \mathrm{C}$ NMR $\delta 195.5$ $(\mathrm{C}=\mathrm{O}), 162.4(\mathrm{C}-8 \mathrm{a}), 158.4(\mathrm{C}-2), 119.6(\mathrm{CN}), 112.7(\mathrm{C}-4 \mathrm{a}), 58.3(\mathrm{C}-3), 49.9(\mathrm{C}-6), 39.8$ (C-8), 39.0 (C-4), 31.7 (C-7), $28.3\left(\mathrm{CH}_{3}\right), 26.7\left(\mathrm{CH}_{3}\right), 144.6,128.2,127.1,126.5$ (Aromatic) Anal. Calcd. for $\mathrm{C}_{18} \mathrm{H}_{18} \mathrm{~N}_{2} \mathrm{O}_{2}$ : C, 73.53; H, 6.16; N, 9.52. Found: C, 73.53; H, $6.31 ; \mathrm{N}, 9.47$.

2-Amino-3-cyano-5,6,7,8-tetrahydro-7,7-dimethyl-4-(2-chlorophenyl)-5-oxo-4Hbenzopyran (4b)

IR (KBr) cm ${ }^{-1} 3469\left(\mathrm{NH}_{2}\right), 2962(\mathrm{C}-\mathrm{H}), 2196(\mathrm{CN}), 1600(\mathrm{C}=\mathrm{C}) ;{ }^{1} \mathrm{H}$ NMR (DMSO), $\delta$ 7.367.38 (dd, 1H, Ar-H), 7.26-7.29 (m, 1H, Ar-H), 7.16-7.22 (m, 2H, Ar-H), $7.03\left(\mathrm{~s}, 2 \mathrm{H}, \mathrm{NH}_{2}\right)$, 4.70 (s, 1H, H-4), 2.53(s, 2H, H-8), 2.27 (d, 1H, H-6), 2.09(d, 1H, H-6'), 1.07 (s, 3H CH 3 ), $0.98\left(\mathrm{~s}, 3 \mathrm{H}, \mathrm{CH}_{3}\right) ;{ }^{13} \mathrm{C}$ NMR $\delta 196.0(\mathrm{C}=\mathrm{O}), 163.6(\mathrm{C}-8 \mathrm{a}), 159.1(\mathrm{C}-2), 119.7(\mathrm{CN}), 112.2$ (C-4a), 57.3 (C-3), 50.4 (C-6), 40.5 (C-8), 39.4 (C-4), $32.3(\mathrm{C}-7), 28.8\left(\mathrm{CH}_{3}\right), 27.3\left(\mathrm{CH}_{3}\right)$, 142.0, 130.4, 129.9, 128.6 (Aromatic) Anal. Calcd. for $\mathrm{C}_{18} \mathrm{H}_{17} \mathrm{ClN}_{2} \mathrm{O}_{2}: \mathrm{C}, 65.85 ; \mathrm{H}, 5.18 ; \mathrm{N}$, 8.54. Found: C, $65.68 ; \mathrm{H}, 5.25 ; \mathrm{N}, 8.49$.

2-Amino-3-cyano-5,6,7,8-tetrahydro-7,7-dimethyl-4-(3-chlorophenyl)-5-oxo-4Hbenzopyran (4c)

IR (KBr) cm ${ }^{-1} 3419\left(\mathrm{NH}_{2}\right), 2961(\mathrm{C}-\mathrm{H}), 2191(\mathrm{CN}), 1660(\mathrm{C}=\mathrm{O}), 1630(\mathrm{C}=\mathrm{C}), 1600(\mathrm{C}=\mathrm{C})$; ${ }^{1} \mathrm{H}$ NMR (DMSO), $\delta$ 7.32-7.35 (m, 1H, Ar-H), 7.26-7.27 (dd, 1H, Ar-H), 7.16-7.17 (m, 1H, Ar-H), 7.10-7.12 (dd, 1H, Ar-H), 7.09 (s, 2H, NH N $_{2}, 4.21$ (s, 1H, H-4), 2.53(s, 2H, H-8), 2.27 (d, 1H, H-6), 2.14 (d, 1H, H-6'), $1.03\left(\mathrm{~s}, 3 \mathrm{H} \mathrm{CH}_{3}\right), 0.96\left(\mathrm{~s}, 3 \mathrm{H}, \mathrm{CH}_{3}\right) ;{ }^{13} \mathrm{C}$ NMR $\delta 196.2$ (C=O), 163.3 (C-8a), 159.0 (C-2), 119.9 (CN), 112.5 (C-4a), 58.0 (C-3), 50.3 (C-6), 40.5 (C-8), 39.4 (C-4), $32.3(\mathrm{C}-7), 28.7\left(\mathrm{CH}_{3}\right), 27.2\left(\mathrm{CH}_{3}\right), 147.6,130.7,127.5,126.4$ (Aromatic). Anal. Calcd. for $\mathrm{C}_{18} \mathrm{H}_{17} \mathrm{ClN}_{2} \mathrm{O}_{2}$ : C, 65.85; H, 5.18; N, 8.54. Found: C, 65.73; H, 5.22; N, 8.60.

\section{2-Amino-3-cyano-5,6,7,8-tetrahydro-7,7-dimethyl-4-(4'-chlorophenyl)-5-oxo- 4H-benzopyran (4d)}

IR (KBr) cm $3380\left(\mathrm{NH}_{2}\right), 2960(\mathrm{C}-\mathrm{H}), 2188(\mathrm{CN}), 1676(\mathrm{C}=\mathrm{O}), 1635(\mathrm{C}=\mathrm{C}), 1603(\mathrm{C}=\mathrm{C}) ;{ }^{1} \mathrm{H}$ NMR (DMSO), 7.15-7.33 (d, 2H), (d, 2H) Ar-H, 7.06 (s, 2H, NH N $_{2}, 4.18$ (s, 1H, H-4), 2.54 (s, 2H, H-8), 2.23 (d, 1H, H-6), 2.14 (d, 1H,H-6'), 1.01 (s, 3H, $\left.\mathrm{CH}_{3}\right), 0.93\left(\mathrm{~s}, 3 \mathrm{H}, \mathrm{CH}_{3}\right) ;{ }^{13} \mathrm{C} \mathrm{NMR} \delta$ $195.5(\mathrm{C}=\mathrm{O}), 163.5(\mathrm{C}-8 \mathrm{a}), 158.4(\mathrm{C}-2), 119.4(\mathrm{CN}), 112.3(\mathrm{C}-4 \mathrm{a}), 57.8$ (C-3), $49.9(\mathrm{C}-6), 39.9$ (C-8), 39.0 (C-4), 31.7 (C-7), $28.2\left(\mathrm{CH}_{3}\right), 26.8\left(\mathrm{CH}_{3}\right), 128.2-131.0$ (Aromatic). Anal. Calcd. for $\mathrm{C}_{18} \mathrm{H}_{17} \mathrm{ClN}_{2} \mathrm{O}_{2}$ : C, 65.85; H, 5.18; N, 8.54. Found: C, 65.75; H, 5.12; N, 8.45.

\section{2-Amino-3-cyano-5,6,7,8-tetrahydro-7,7-dimethyl-4-(2-nitrophenyl)-5-oxo-4H- benzopyran (4e)}

IR (KBr) cm ${ }^{-1} 3471\left(\mathrm{NH}_{2}\right), 3076(\mathrm{C}-\mathrm{H}), 2194(\mathrm{CN}), 1688(\mathrm{C}=\mathrm{O}), 1665(\mathrm{C}=\mathrm{C}), 1597(\mathrm{C}=\mathrm{C})$, $1526\left(\mathrm{NO}_{2}\right) ;{ }^{1} \mathrm{H}$ NMR (DMSO), $\delta$ 7.80-7.83 (dd, 1H, Ar-H), 7.64-7.68 (m, 1H, Ar-H), 7.41-7.44 (m, 1H, Ar-H), 7.35-7.37 (dd, 1H, Ar-H), 7.19 (s, 2H, NH $), 4.94$ (s, 1H, H-4), 2.55 (s, 2H, $\mathrm{H}-8), 2.22$ (d, 1H, H-6), 2.03 (d, 1H, H-6'), $1.01\left(\mathrm{~s}, 3 \mathrm{H}, \mathrm{CH}_{3}\right), 0.88\left(\mathrm{~s}, 3 \mathrm{H}, \mathrm{CH}_{3}\right) ;{ }^{13} \mathrm{C} \mathrm{NMR} \delta$ $196.3(\mathrm{C}=\mathrm{O}), 163.2(\mathrm{C}-8 \mathrm{a}), 159.6(\mathrm{C}-2), 119.5(\mathrm{CN}), 112.7$ (C-4a), $56.8(\mathrm{C}-3), 50.0(\mathrm{C}-6), 40.5$ (C-8), 39.4 (C-4), 32.3 (C-7), $28.7\left(\mathrm{CH}_{3}\right), 27.1\left(\mathrm{CH}_{3}\right), 149.4,130.7,128.3,124.1$ (Aromatic). Anal. Calcd. for $\mathrm{C}_{18} \mathrm{H}_{17} \mathrm{~N}_{3} \mathrm{O}_{4}$ : C, 63.72; H, 5.01; N, 8.26. Found: C,63.90; H, 5.10; N, 8.32. 
2-Amino-3-cyano-5,6,7,8-tetrahydro-7,7-dimethyl-4-(3-nitrophenyl)-5-oxo-4Hbenzopyran (4f)

IR $(\mathrm{KBr}) \mathrm{cm}^{-1} 3430\left(\mathrm{NH}_{2}\right), 3101(\mathrm{C}-\mathrm{H}), 2187(\mathrm{CN}), 1654(\mathrm{C}=\mathrm{O}), 1597(\mathrm{C}=\mathrm{C}), 1531\left(\mathrm{NO}_{2}\right)$; ${ }^{1} \mathrm{H}$ NMR (DMSO), $\delta$ 8.07-8.09 (m, 1H, Ar-H), 7.98-7.99 (m, 1H, Ar-H), 7.66-7.68 (m, 1H, Ar-H), 7.61-7.64 (m, 1H, Ar-H), 7.18 (s, 2H, NH $\mathrm{NH}_{2}, 4.42$ (s, 1H, H-4), 2.55 (s, 2H, H-8), 2.28 (d, 1H, H-6), 2.13 (d, 1H, H-6'), $1.04\left(\mathrm{~s}, 3 \mathrm{H}, \mathrm{CH}_{3}\right), 0.96\left(\mathrm{~s}, 3 \mathrm{H}, \mathrm{CH}_{3}\right) ;{ }^{13} \mathrm{C}$ NMR $\delta 196.2$ $(\mathrm{C}=\mathrm{O}), 163.6(\mathrm{C}-8 \mathrm{a}), 159.1(\mathrm{C}-2), 119.8(\mathrm{CN}), 112.2$ (C-4a), 57.6 (C-3), 50.3 (C-6), 40.4 (C-8), 39.4 (C-4), 32.2 (C-7), $28.7\left(\mathrm{CH}_{3}\right), 27.2\left(\mathrm{CH}_{3}\right), 148.2$, 134.6, 130.4, 122.2 (Aromatic). Anal. Calcd. for $\mathrm{C}_{18} \mathrm{H}_{17} \mathrm{~N}_{3} \mathrm{O}_{4}$ : C, 63.72; H, 5.01; N, 8.26. Found: C, 63.85; H, 5.13; N, 8.19.

2-Amino-3-cyano-5,6,7,8-tetrahydro-7,7-dimethyl-4-(4'-nitrophenyl)-5-oxo-4Hbenzopyran $(4 \mathrm{~g})$

IR $(\mathrm{KBr}) \mathrm{cm}^{-1} 3407\left(\mathrm{NH}_{2}\right), 2970(\mathrm{C}-\mathrm{H}), 2184(\mathrm{CN}), 1671(\mathrm{C}=\mathrm{O}), 1630(\mathrm{C}=\mathrm{C}), 1594(\mathrm{C}=\mathrm{C})$, $1520\left(\mathrm{NO}_{2}\right) ;{ }^{1} \mathrm{H}$ NMR (DMSO), $\delta$ 8.16-8.18 (m, 2H, Ar-H), 7.44-7.46 (m, 2H, Ar-H), 7.18 (s, 2H, NH $\mathrm{NH}_{2}, 4.36$ (s, 1H, H-4), 2.57 (s, 2H, H-8), 2.28 (d, 1H, H-6), 2.13 (d, 1H, H-6'), 1.04 $\left(\mathrm{s}, 3 \mathrm{H} \mathrm{CH}_{3}\right), 0.95\left(\mathrm{~s}, 3 \mathrm{H}, \mathrm{CH}_{3}\right) ;{ }^{13} \mathrm{C}$ NMR $\delta 196.2(\mathrm{C}=\mathrm{O}), 163.5(\mathrm{C}-8 \mathrm{a}), 159.0(\mathrm{C}-2), 119.7$ (CN), 112.1 (C-4a), 57.4 (C-3), 50.3 (C-6), 40.4 (C-8), 39.4 (C-4), 32.2 (C-7), $28.7\left(\mathrm{CH}_{3}\right)$, $27.4\left(\mathrm{CH}_{3}\right), 146.7,129.0,124.1$ (Aromatic). Anal. Calcd. for $\mathrm{C}_{18} \mathrm{H}_{17} \mathrm{~N}_{3} \mathrm{O}_{4}: \mathrm{C}, 63.72 ; \mathrm{H}, 5.01$; N, 8.26. Found: C, 63.60; H, 5.08; N, 8.19.

\section{2-Amino-3-cyano-5,6,7,8-tetrahydro-7,7-dimethyl-4-(4'-methylphenyl)-5-oxo- 4 H-benzopyran (4h)}

IR $(\mathrm{KBr}) \mathrm{cm}^{-1} 3426\left(\mathrm{NH}_{2}\right), 2956(\mathrm{C}-\mathrm{H}), 2191(\mathrm{CN}), 1682(\mathrm{C}=\mathrm{O}), 1639(\mathrm{C}=\mathrm{C}), 1601(\mathrm{C}=\mathrm{C})$; ${ }^{1} \mathrm{H}$ NMR (DMSO), $\delta$ 7.08-7.10 (d, 2H, Ar-H), 7.02-7.03 (d, 2H, Ar-H), $6.96\left(\mathrm{~s}, 2 \mathrm{H}, \mathrm{NH}_{2}\right)$, 4.13 (s, 1H, H-4), 2.51 (s, 2H, H-8), $2.24\left(\mathrm{~s}, 3 \mathrm{H}, \mathrm{CH}_{3}\right), 2.26$ (d, 1H, H-6), 2.10 (d, 1H, H-6'), $1.03\left(\mathrm{~s}, 3 \mathrm{H}, \mathrm{CH}_{3}\right), 0.95\left(\mathrm{~s}, 3 \mathrm{H}, \mathrm{CH}_{3}\right) ;{ }^{13} \mathrm{C}$ NMR $\delta 196.1(\mathrm{C}=\mathrm{O}), 162.7(\mathrm{C}-8 \mathrm{a}), 158.9(\mathrm{C}-2)$, $120.2(\mathrm{CN}), 113.3$ (C-4a), 58.9 (C-3), 50.4 (C-6), 40.5 (C-8), 39.4 (C-4), 32.2 (C-7), 28.8 $\left(\mathrm{CH}_{3}\right), 27.2\left(\mathrm{CH}_{3}\right), 21.0\left(\mathrm{CH}_{3}, \mathrm{Aryl}\right), 142.2,136.1,129.3,127.5$ (Aromatic). Anal. Calcd. for $\mathrm{C}_{19} \mathrm{H}_{20} \mathrm{~N}_{2} \mathrm{O}_{2}$ : C, 74.03; H, 6.49; N, 9.09. Found: C,74.23; H, 6.55; N, 9.17.

2-Amino-3-cyano-5,6,7,8-tetrahydro-7,7-dimethyl-4-(4'-N,N-dimethylaminophenyl)5-oxo-4H-benzopyran (4i)

IR $(\mathrm{KBr}) \mathrm{cm}^{-1} 3382\left(\mathrm{NH}_{2}\right), 2959(\mathrm{C}-\mathrm{H}), 2191(\mathrm{CN}), 1687(\mathrm{C}=\mathrm{O}), 1655(\mathrm{C}=\mathrm{C}), 1608(\mathrm{C}=\mathrm{C})$; ${ }^{1} \mathrm{H}$ NMR (DMSO), $\delta$ 6.93-6.95 (d, 2H, Ar-H), 6.62-6.64 (d, 2H, Ar-H), $6.88\left(\mathrm{~s}, 2 \mathrm{H}, \mathrm{NH}_{2}\right)$, 4.04 (s, 1H, H-4), $2.84\left(\mathrm{~s}, 6 \mathrm{H},\left(\mathrm{CH}_{3}\right)_{2}\right), 2.53$ (s, 2H, H-8), 2.25 (d, 1H, H-6), 2.09 (d, 1H, H6), $1.06\left(\mathrm{~s}, 3 \mathrm{H}, \mathrm{CH}_{3}\right), 0.95\left(\mathrm{~s}, 3 \mathrm{H}, \mathrm{CH}_{3}\right) ;{ }^{13} \mathrm{C}$ NMR $\delta 196.1(\mathrm{C}=\mathrm{O}), 162.3(\mathrm{C}-8 \mathrm{a}), 158.8(\mathrm{C}-$ 2), $120.4(\mathrm{CN}), 112.8$ (C-4a), 59.4 (C-3), 50.5 (C-6), 40.6 (C-8), 39.9 (C-4), 32.2 (C-7), 28.9 $\left(\mathrm{CH}_{3}\right), 27.2\left(\mathrm{CH}_{3}\right), \mathrm{N}\left(\mathrm{CH}_{3}\right)_{2}$ merged with solvent peaks, 149.7, 132.9, 128.1 (Aromatic). Anal. Calcd. for $\mathrm{C}_{20} \mathrm{H}_{23} \mathrm{~N}_{3} \mathrm{O}_{2}$ : C, 71.22; H, 6.82; N, 8.31. Found: C, 71.03; H, 6.90; N, 8.23.

2-Amino-3-cyano-5,6,7,8-tetrahydro-7,7-dimethyl-4-(4'-methoxyphenyl)-5-oxo-4Hbenzopyran $(4 j)$

IR $(\mathrm{KBr}) \mathrm{cm}^{-1} 3375\left(\mathrm{NH}_{2}\right), 2962(\mathrm{C}-\mathrm{H}), 2193(\mathrm{CN}), 1683(\mathrm{C}=\mathrm{O}), 1655(\mathrm{C}=\mathrm{C}), 1605(\mathrm{C}=\mathrm{C})$; ${ }^{1} \mathrm{H}$ NMR (DMSO), $\delta$ 6.82-6.84 (d, 2H, Ar-H), 7.01-7.04 (d, 2H, Ar-H), 6.97 (s, 2H, NH $\mathrm{N}_{2}$ ), $4.11(\mathrm{~s}, 1 \mathrm{H}, \mathrm{H}-4), 3.71\left(\mathrm{~s}, 3 \mathrm{H}, \mathrm{OCH}_{3}\right), 2.54(\mathrm{~s}, 2 \mathrm{H}, \mathrm{H}-8), 2.24(\mathrm{~d}, 1 \mathrm{H}, \mathrm{H}-6), 2.08(\mathrm{~d}, 1 \mathrm{H}, \mathrm{H}-$ 6), $1.04\left(\mathrm{~s}, 3 \mathrm{H}, \mathrm{CH}_{3}\right), 0.97\left(\mathrm{~s}, 3 \mathrm{H}, \mathrm{CH}_{3}\right) ;{ }^{13} \mathrm{C}$ NMR $\delta 195.6(\mathrm{C}=\mathrm{O}), 162.0(\mathrm{C}-8 \mathrm{a}), 158.4(\mathrm{C}-$ 2), $119.7(\mathrm{CN}), 113.6$ (C-4a), 58.6 (C-3), 50.0 (C-6), 39.9 (C-8), 39.1 (C-4), 31.7 (C-7), 28.3 
$\left(\mathrm{CH}_{3}\right), 26.7\left(\mathrm{CH}_{3}\right), 54.9\left(\mathrm{OCH}_{3}, \mathrm{Aryl}\right), 136.8,128.1,113.0$ (Aromatic). Anal. Calcd. for $\mathrm{C}_{19} \mathrm{H}_{20} \mathrm{~N}_{2} \mathrm{O}_{3}$ : C, 70.37; H, 6.17; N, 8.64. Found: C, 70.20; H, 6.09; N, 8.72.

2-Amino-3-cyano-5,6,7,8-tetrahydro-7,7-dimethyl-4-(3,4-dimethoxyphenyl)-5-oxo4 H-benzopyran (4k)

IR $(\mathrm{KBr}) \mathrm{cm}^{-1} 3390\left(\mathrm{NH}_{2}\right), 2955(\mathrm{C}-\mathrm{H}), 2193(\mathrm{CN}), 1682(\mathrm{C}=\mathrm{O}), 1658(\mathrm{C}=\mathrm{C}), 1603(\mathrm{C}=\mathrm{C}) ;{ }^{1} \mathrm{H}$ NMR (DMSO), $\delta$ 6.86-6.88 (d,1H, Ar-H), 6.69-6.70 (d, 1H, Ar-H), 6.64-6.67 (dd, 1H, Ar-H), $3.71\left(\mathrm{~d}, 6 \mathrm{H},\left(\mathrm{OCH}_{3}\right)_{2}\right), 6.95\left(\mathrm{~s}, 2 \mathrm{H}, \mathrm{NH}_{2}\right), 4.13(\mathrm{~s}, 1 \mathrm{H}, \mathrm{H}-4), 2.50(\mathrm{~s}, 2 \mathrm{H}, \mathrm{H}-8), 2.24(\mathrm{~d}, 1 \mathrm{H}, \mathrm{H}-$ 6), 2.12 (d, 1H, H-6'), $1.04\left(\mathrm{~s}, 3 \mathrm{H}, \mathrm{CH}_{3}\right), 0.97\left(\mathrm{~s}, 3 \mathrm{H}, \mathrm{CH}_{3}\right) ;{ }^{13} \mathrm{C} \mathrm{NMR} \delta 196.1(\mathrm{C}=\mathrm{O}), 162.7$ (C8a), 158.8 (C-2), 119.5 (CN), 112.2 (C-4a), 59.0 (C-3), 50.4 (C-6), 40.5 (C-8), 39.9 (C-4), 32.2 (C-7), $28.9\left(\mathrm{CH}_{3}\right), 27.1\left(\mathrm{CH}_{3}\right)$, 55.96, $55.92\left(\mathrm{OCH}_{3}\right.$, Aryl), 148.9,137.7, 113.3 (Aromatic). Anal. Calcd for $\mathrm{C}_{20} \mathrm{H}_{23} \mathrm{~N}_{2} \mathrm{O}_{4}$ : C, 67.81; H, 6.48; N, 7.89. Found: C, 67.72; H, 6.40; N, 7.81.

2-Amino-3-cyano-5,6,7,8-tetrahydro-7,7-dimethyl-4-(3-bromophenyl)-5-oxo-4Hbenzopyran (4l)

IR (KBr) cm $\mathrm{cm}^{-1} 3343\left(\mathrm{NH}_{2}\right), 2962(\mathrm{C}-\mathrm{H}), 2191(\mathrm{CN}), 1687(\mathrm{C}=\mathrm{O}), 1657(\mathrm{C}=\mathrm{C}), 1603(\mathrm{C}=\mathrm{C})$; ${ }^{1} \mathrm{H}$ NMR (DMSO), $\delta$ 7.38-7.41 (m, 1H, Ar-H), 7.30-7.31 (m, 1H, Ar-H), 7.26-7.29 (m, 1H, Ar-H), 7.15-7.17 (m, 1H, Ar-H), 7.09 (s, 2H, NH 2$), 4.20$ (s, 1H, H-4), 2.53 (s, 2H, H-8), 2.24 (d, 1H, H-6), 2.11 (d, 1H, H-6'), $1.03\left(\mathrm{~s}, 3 \mathrm{H}, \mathrm{CH}_{3}\right), 0.96$ (s, 3H, $\left.\mathrm{CH}_{3}\right) ;{ }^{13} \mathrm{C}$ NMR $\delta 196.2$ $(\mathrm{C}=\mathrm{O}), 163.3(\mathrm{C}-8 \mathrm{a}), 159.0(\mathrm{C}-2), 119.6(\mathrm{CN}), 112.5$ (C-4a), 58.1 (C-3), 50.3 (C-6), 40.5 (C-8), 39.4 (C-4), $32.3(\mathrm{C}-7), 28.7\left(\mathrm{CH}_{3}\right), 27.2\left(\mathrm{CH}_{3}\right), 147.9,131.1,129.9,126.8$ (Aromatic). Anal. Calcd. for $\mathrm{C}_{18} \mathrm{H}_{17} \mathrm{BrN}_{2} \mathrm{O}_{2}$ : C, 57.91; H, 4.56; N, 7.51. Found: C, 57.78; H, 4.49; N, 7.62.

2-Amino-3-cyano-5,6,7,8-tetrahydro-7,7-dimethyl-4-(4'-fluorophenyl)-5-oxo-4Hbenzopyran $(4 m)$

IR (KBr) cm ${ }^{-1} 3343\left(\mathrm{NH}_{2}\right), 2962(\mathrm{C}-\mathrm{H}), 2191(\mathrm{CN}), 1687(\mathrm{C}=\mathrm{O}), 1657(\mathrm{C}=\mathrm{C}), 1603(\mathrm{C}=\mathrm{C})$; ${ }^{1} \mathrm{H}$ NMR (DMSO), $\delta$ 7.17-7.20 (m, 2H, Ar-H), 7.09-7.13 (m, 2H, Ar-H), 7.03 (s, 2H, NH $\mathrm{NH}_{2}$, 4.20 (s, 1H, H-4), 2.51 (s, 2H, H-8), 2.26 (d, 1H, H-6), 2.12 (d, 1H, H-6'), 1.03 (s, 3H, CH CH $_{3}$, $0.94\left(\mathrm{~s}, 3 \mathrm{H}, \mathrm{CH}_{3}\right) ;{ }^{13} \mathrm{C}$ NMR $\delta 196.1(\mathrm{C}=\mathrm{O}), 162.9(\mathrm{C}-8 \mathrm{a}), 158.9(\mathrm{C}-2), 120.1(\mathrm{CN}), 113.0$ (C-4a), 58.5 (C-3), 50.4 (C-6), 40.4 (C-8), 39.4 (C-4), $32.2(\mathrm{C}-7), 28.7\left(\mathrm{CH}_{3}\right), 27.3(\mathrm{CH} 3)$, 141.1, 129.5, 115 (Aromatic). Anal. Calcd. for $\mathrm{C}_{18} \mathrm{H}_{17} \mathrm{FN}_{2} \mathrm{O}_{2:} \mathrm{C}, 69.23 ; \mathrm{H}, 5.45 ; \mathrm{N}, 8.97$. Found; C, 69.35; H, 5.38; N, 8.88.

2-Amino-3-cyano-5,6,7,8-tetrahydro-7,7-dimethyl-4-(Phenyl allylidene)-5-oxo-4Hbenzopyran $(4 n)$

IR $(\mathrm{KBr}) \mathrm{cm}^{-1} 3386\left(\mathrm{NH}_{2}\right), 2962(\mathrm{C}-\mathrm{H}), 2186(\mathrm{CN}), 1648(\mathrm{C}=\mathrm{O}), 1604(\mathrm{C}=\mathrm{C}) ;{ }^{1} \mathrm{H}$ NMR (DMSO), $\delta$ 7.37-7.39 (d, 2H, Ar-H), 7.29-7.31 (m, 2H, Ar-H), 7.20-7.23 (m, 1H, Ar-H), $7.06\left(\mathrm{~s}, 2 \mathrm{H}, \mathrm{NH}_{2}\right), 6.35,6.10(2 \mathrm{H}, \mathrm{CH}=\mathrm{CH}), 3.83$ (s, 1H, H-4), 2.51 (s, 2H, H-8), 2.29 (d, $1 \mathrm{H}, \mathrm{H}-6), 2.23$ (d, 1H, H-6'), $1.03\left(\mathrm{~s}, 3 \mathrm{H}, \mathrm{CH}_{3}\right), 1.00\left(\mathrm{~s}, 3 \mathrm{H}, \mathrm{CH}_{3}\right) ;{ }^{13} \mathrm{C}$ NMR $\delta 196.3(\mathrm{C}=\mathrm{O})$, 162.9 (C-8a), 159.7 (C-2), 120.3 (CN), 112.3 (C-4a), 55.6 (C-3), 50.5 (C-6), 40.5 (C-8), 39.4 (C-4), 32.2 (C-7), $28.6\left(\mathrm{CH}_{3}\right), 27.4\left(\mathrm{CH}_{3}\right), 136.9,131.5,129.7,126.6$ (Aromatic). Anal. Calcd. for $\mathrm{C}_{20} \mathrm{H}_{20} \mathrm{~N}_{2} \mathrm{O}_{2}$ : C, 75.01; H, 6.25; N, 8.75. Found: C, 75.17; H, 6.17; N, 8.82.

2-Amino-3-cyano-5,6,7,8-tetrahydro-7,7-dimethyl-4-(2-furfuryl)-5-oxo-4Hbenzopyran (4o)

IR (KBr) cm ${ }^{-1} 3396\left(\mathrm{NH}_{2}\right), 2966(\mathrm{C}-\mathrm{H}), 2196(\mathrm{CN}), 1680(\mathrm{C}=\mathrm{O}), 1660(\mathrm{C}=\mathrm{C}), 1603(\mathrm{C}=\mathrm{C})$; ${ }^{1} \mathrm{H}$ NMR (DMSO), $\delta 7.48$ (s, 1H, Ar-H), 6.32-6.33 (dd, 1H, Ar-H), 6.05 (d, 1H, Ar-H), 7.08 (s, 2H, NH $\mathrm{NH}_{2}, 4.33$ (s, 1H, H-4), 2.50 (s, 2H, H-8), 2.30 (d, 1H, H-6), 2.15 (d, 1H, H-6'), 1.04 $\left(\mathrm{s}, 3 \mathrm{H}, \mathrm{CH}_{3}\right), 0.99\left(\mathrm{~s}, 3 \mathrm{H}, \mathrm{CH}_{3}\right),{ }^{13} \mathrm{C}$ NMR $\delta 195.9(\mathrm{C}=\mathrm{O}), 163.7(\mathrm{C}-8 \mathrm{a}), 159.7(\mathrm{C}-2), 120.0(\mathrm{CN})$, 
110.8 (C-4a), 55.8 (C-3), 50.3 (C-6), 40.4 (C-8), 39.4 (C-4), 32.2 (C-7), $28.8\left(\mathrm{CH}_{3}\right), 27.0$ $\left(\mathrm{CH}_{3}\right), 156.1,142.2,110.9,105.5$ (Aromatic). Anal. Calcd. for $\mathrm{C}_{17} \mathrm{H}_{17} \mathrm{~N}_{2} \mathrm{O}_{3}: \mathrm{C}, 68.69 ; \mathrm{H}$, 5.72; N, 9.43. Found: C, 68.81; H, 5.80; N, 9.50.

\section{Results and Discussion}

A mixture of benzaldehyde, malononitrile and 5,5-dimethyl-1,3-cyclohexanedione (dimedone) were treated with tetrabutylammoniumbromide under three different conditions, namely (i) using ethanol as solvent (method A); (ii) using water as reaction medium (method B); (iii) grinding in a mortar with pestle (method C) at room temperature, to give desired product derivatives (Scheme 1). To explore the scope of this reaction, we extended the reaction to various aromatic aldehydes. The reaction time, yield and the physical constants of various products obtained by the reaction are given in Table 1.

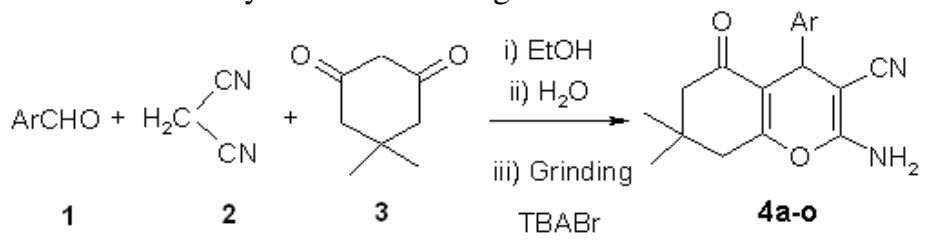

\section{Scheme 1}

The yields are, in general, very high regardless of the structural variations in aromatic aldehyde, but show obvious effects in terms of reaction time, under these reaction conditions. Aromatic aldehydes containing electron-donating groups (such as alkoxy group, methyl group and dimethylamino group, $\mathbf{4 h - k}$ in table 1) have taken longer reaction time, whereas the reaction time is less for the aldehyde containing electron-withdrawing groups (such as nitro or halide, $\mathbf{4 b - g}$ and $\mathbf{4 l - m}$ in table 1 ).

The catalyst plays a crucial role in the success of the reaction in terms of the rate and the yields. For example, benzaldehyde reacts with malonitrile and dimedone in the presence of $1 \mathrm{~mol} \% \mathrm{TBABr}$ in ethanol giving the product $4 \mathbf{a}$ of $70 \%$ yield. Increasing the percentage of catalyst to $5 \mathrm{~mol} \%$ and $10 \mathrm{~mol} \%$ results the yields $82 \%$ and $92 \%$ respectively. Higher amounts of the catalyst did not improve the results to a greater extent. Thus, $10 \mathrm{~mol} \%$ $\mathrm{TBABr}$ was chosen as the maximum quantity of catalyst used for these reactions. We performed the reaction in ethanol without the catalyst and isolated the cyano olefin intermediate and unreacted $\mathbf{3}$.

A mechanism for the reaction is outlined in Scheme 2. The reaction proceeds via initial formation of cyanoolefin [A] formed by the condensation of aryl aldehyde $\mathbf{1}$ and malononitrile 2.Cyanoolefin [A] reacts with the active methylene moiety of $\mathbf{3}$ giving intermediate $[\mathrm{B}]$ which subsequently cyclises to afford the desired compound $\mathbf{4}$. The water molecule eliminated in the first step plays a key role in the cyclisation process.

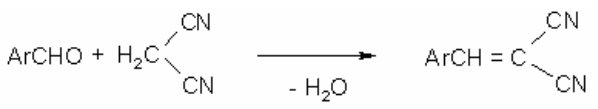

[A]

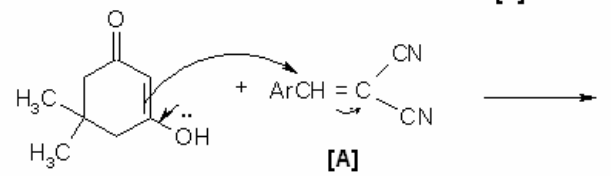




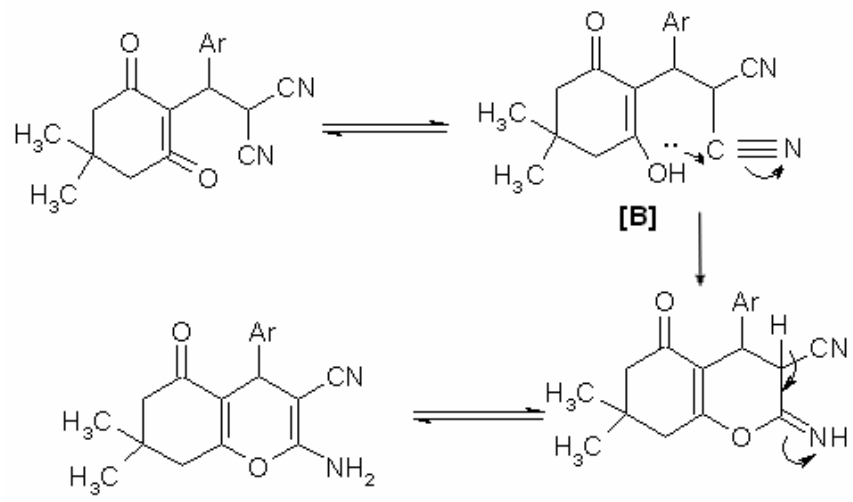

\section{Conclusion}

Scheme 2

We have described a general and highly efficient procedure for the preparation of tetrahydrobenzo $[b]$ pyrans catalysed by $\mathrm{TBABr}$ three component condensation under three different conditions. Among the above methods, the solvent-free procedure (method $\mathrm{C}$ ) provided the best results. Although the reaction carried out in water (method B) showed similar results as in ethanol (method A), they are less expensive and less toxic than those with organic solvents. Compared to other methods using $\mathrm{TBABr}$ as catalyst, has several advantages including high yields, inexpensive operation and minimal environmental impact which make it a useful process for the synthesis of these compounds.

\section{Acknowledgement}

The authors are thankful to the Head of the Department of Chemistry, authorities of the University for providing necessary facilities to complete this work and to the Indian Institute of Technology, Chennai for recording the spectra.

\section{References}

1. Green G R, Evans J M and Vong A K, In comprehensive Hetrocyclic Chemistry II; Katritzky A R, Rees C W and Scriven E F V Eds.; Pergamon Press: Oxford, 1995, 5, 469.

2. Armesto D, Horspool W M, Martin N, Ramos A and Seaone C, J Org Chem., 1989, 54, 3069.

3. Hatakeyama S, Ochi N, Numata H and Takano S, J Chem Soc Commun., 1988, 1202.

4. Devi I and Bhuyan P J, Tetrahedron Lett., 2004, 45, 8625-8627.

5. Fotouhi L, Heravi M.M, Fatechi A and Bakhtiari K, Tetrahedron Lett., 2007, 48, 5379-5381

6. Jin T S, Wang A Q, Wang X, Zhang J S and Li T S, Synlett., 2004, 871-873.

7. Shi D, Mou J, Zhuang Q and Wang X, J Chem Res., 2004, 821.

8. Balalaie S, Bararjanian M, Amani A M and Movassagh B, Synlett., 2006, 263-266.

9. Wang L M, Shao Jh, Tian H, Wang Y H and Liu B, J Fluorine Chem., 2006, 127, 97.

10. Lian X Z, Huang Y, Li Y Q and Zheng J W, Monatshefte fur Chemie, 2007, 1007.

11. Jin T S, Xiao J C, Wang S J, Li T S and Song X R, Synlett., 2003, 2001-2004.

12. Sharanin Y A, Promonenkov V K and Sharanina L G, J Org Chem., 1982, 18, 544-548.

13. Wang X S, Shi D Q, Tu S J and Yao C S, Syn Commun., 2003, 33(1), 119. 


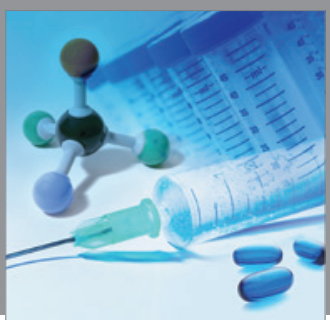

International Journal of

Medicinal Chemistry

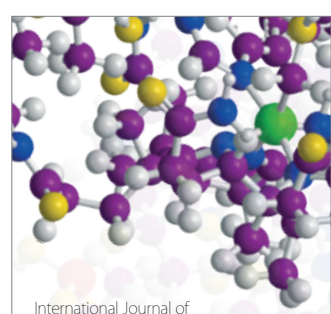

Carbohydrate Chemistry

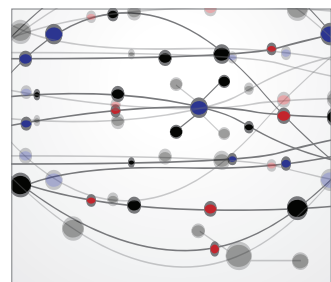

The Scientific World Journal
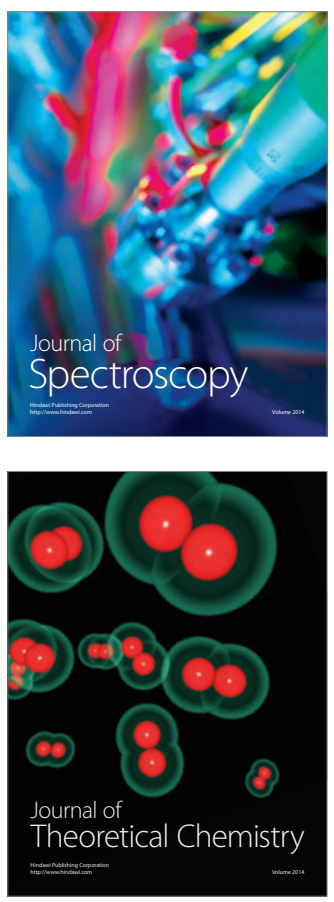
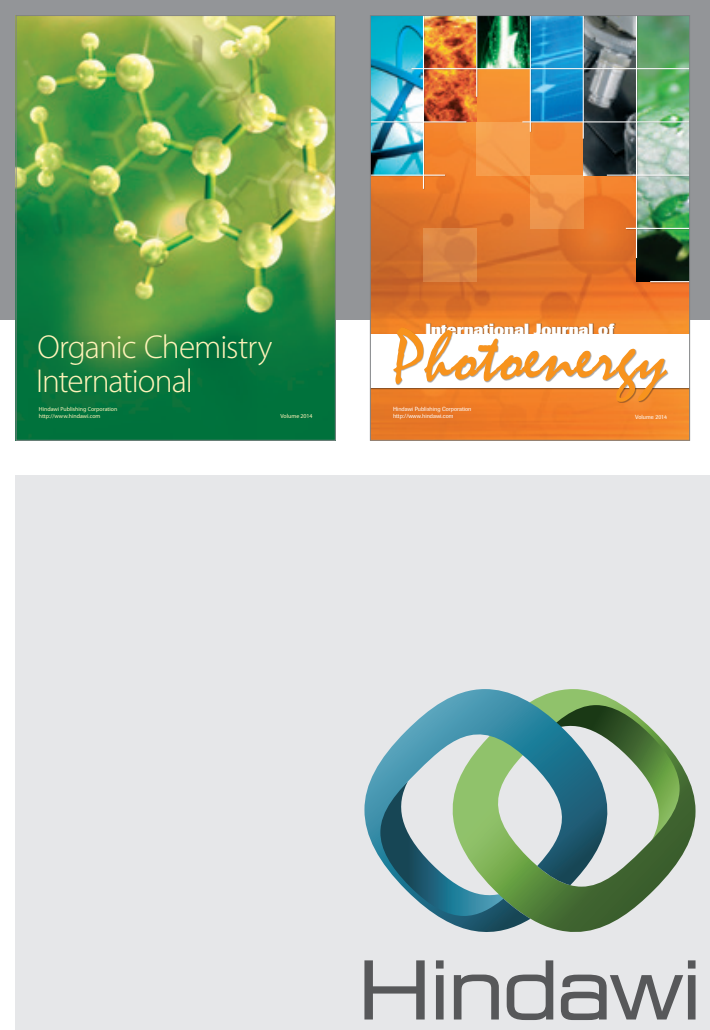

Submit your manuscripts at

http://www.hindawi.com
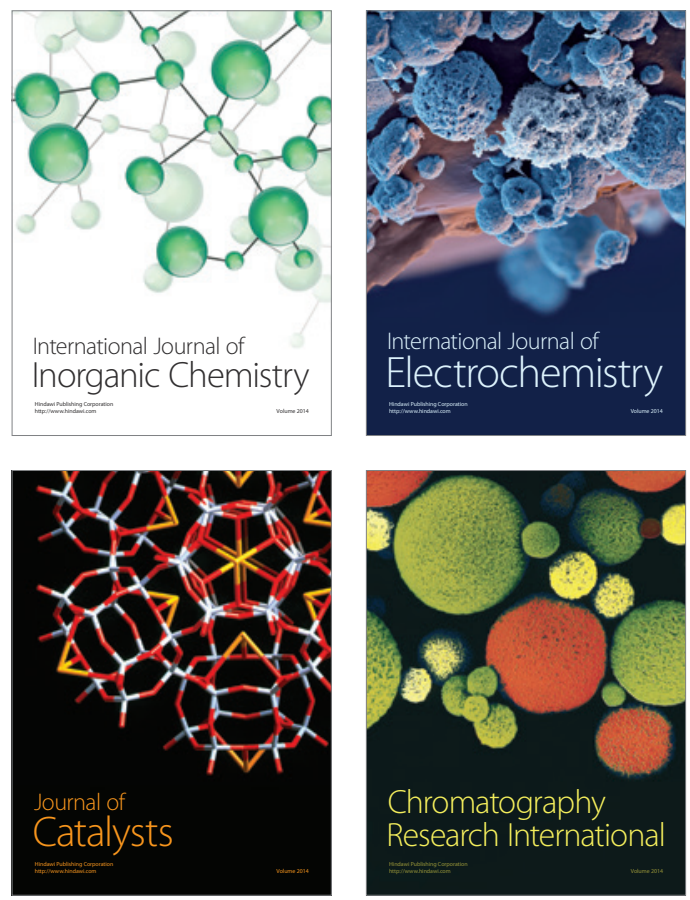
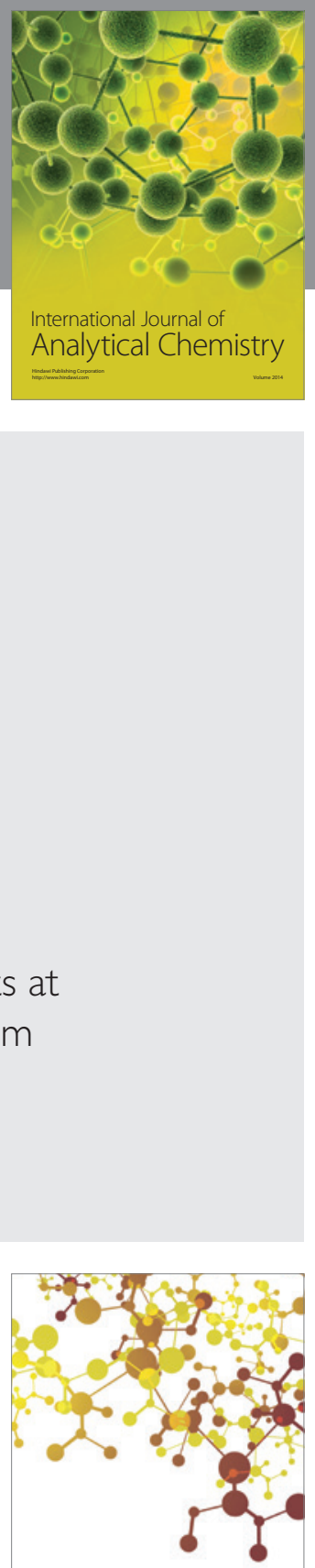

Journal of

Applied Chemistry
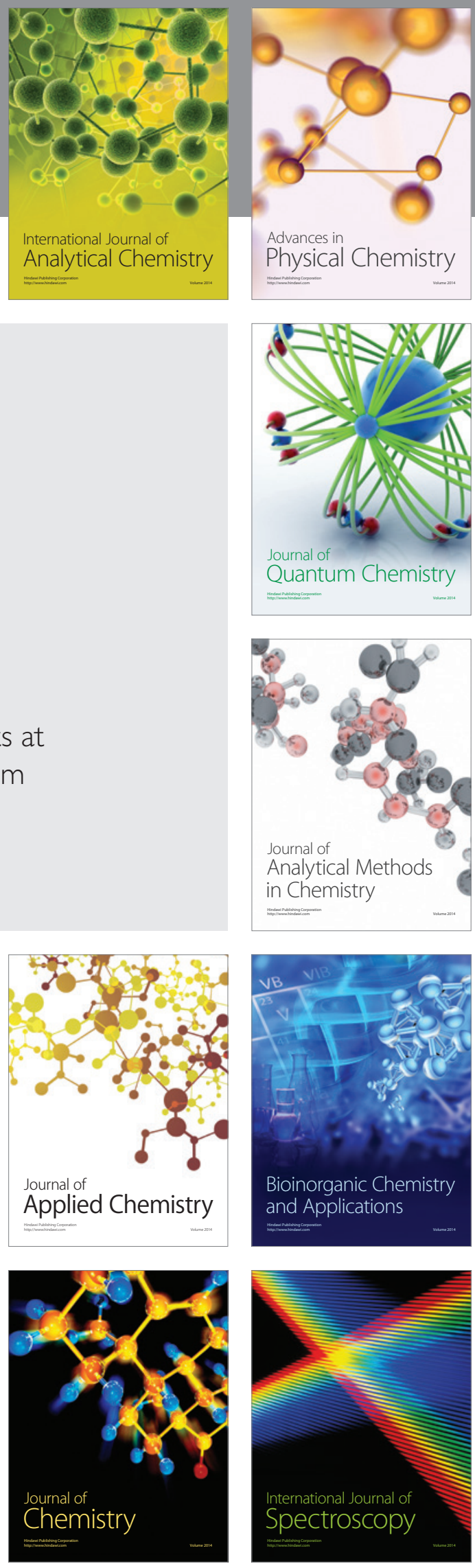